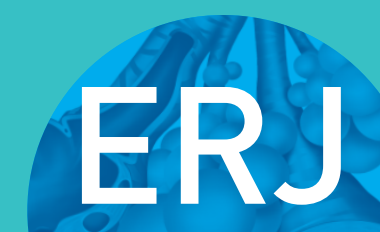

open research
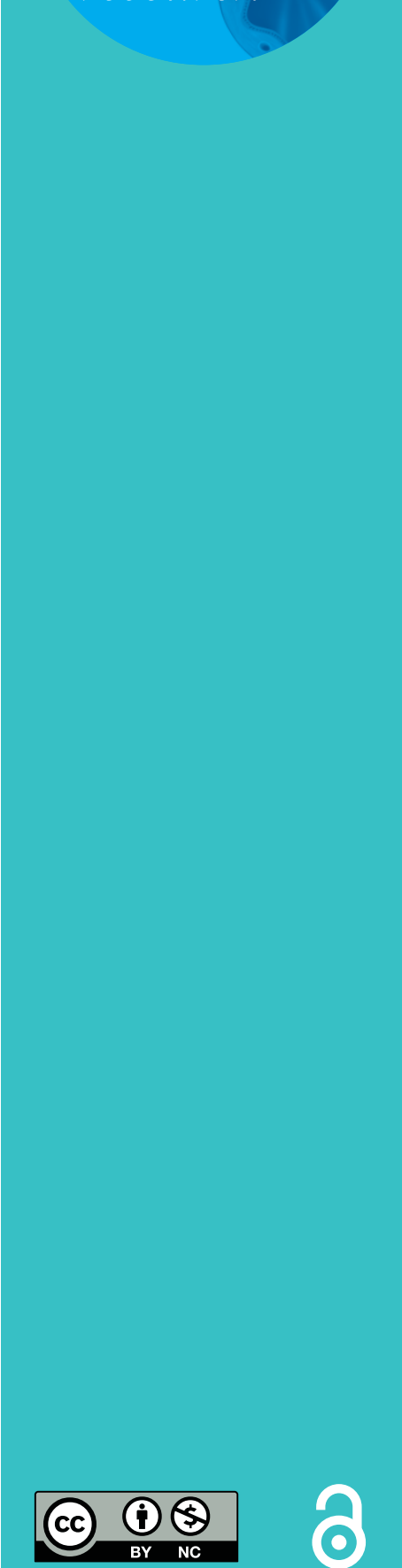

\section{The impact of a dedicated physiotherapist clinic for children with dysfunctional breathing}

\author{
Nicola J. Barker $\mathbb{1}^{1}$, Heather Elphick ${ }^{1}$ and Mark L. Everard ${ }^{2}$
}

Affiliations: ${ }^{1}$ Dept of Respiratory Medicine, Sheffield Children's Hospital, Sheffield, UK. ${ }^{2}$ School of Paediatrics and Child Health, University of Western Australia, TelethonKids Institute, Princess Margaret Hospital for Children, Subiaco, Australia.

Correspondence: Nicola J. Barker, Physiotherapy Dept, Sheffield Children's Hospital, Western Bank, Sheffield S10 2TH, UK. E-mail: nicki.barkerdasch.nhs.uk

ABSTRACT Dysfunctional breathing is a significant cause of morbidity, adversely affecting an individual's quality of life. There is currently no data from paediatric centres on the impact of breathing retraining for dysfunctional breathing.

Symptoms and quality of life were measured in 34 subjects referred sequentially for breathing retraining to the first dedicated paediatric dysfunctional breathing clinic in the UK. Data were obtained prior to the first intervention (time point 1), at discharge (time point 2) and by post 6 months later (time point 3).

The mean (interquartile range) age of participants was 13.3 (9.1-16.3) years, with $52 \%$ female. Data were obtained at time points 2 and 3 in 23 and 13 subjects, respectively.

Statistically significant improvements were observed in symptom scores, child quality of life and parental proxy quality of life between time points 1 and $2(p<0.0001)$, while there was no significant difference in the data at time point 3 as compared with time point 2.

This study suggests that physiotherapist-led breathing retraining offers significant benefit to young people with dysfunctional breathing which is maintained for at least 6 months after treatment is completed. Future studies will provide more information on the long-term effects of interventions for dysfunctional breathing.

@ERSpublications

Breathing retraining improves the quality of life and symptoms of children with dysfunctional breathing http://ow.ly/qg1c302sYAF

Received: Dec 222015 | Accepted after revision: June 252016

Conflict of interest: None declared

Copyright $\odot$ ERS 2016. This article is open access and distributed under the terms of the Creative Commons Attribution Non-Commercial Licence 4.0. 


\section{Introduction}

Dysfunctional breathing affects children (and adults) and is primarily characterised by abnormal breathing patterns [1-5]. These patterns most commonly present as excessive upper chest and accessory muscle activity with the diaphragm contributing little to changes in thoracic volume. For others, an upper airway component may be most prominent, as seen in subjects with paradoxical vocal cord dysfunction [6, 7], but in these subjects it appears that the thoracic disordered breathing is still present and that they form a part of the continuum of habituated responses adversely affecting the normal pattern of respiration. Estimates of prevalence of dysfunctional breathing are problematic due to the lack of a simple diagnostic test, but it has been estimated to affect $8 \%$ of the adult population and $30 \%$ of adults with asthma [8, 9]. One questionnaire study suggested that $5.3 \%$ of children aged 5-18 years with asthma experienced dysfunctional breathing as an important comorbidity [10], although this is likely to be an underestimate.

In children and adolescents the most common symptoms of dysfunctional breathing are breathlessness (at rest or with exercise), chest tightness, chest pain, deep sighing and frequent yawning $[1,2]$. These symptoms are usually episodic, although they can be persistent. They are often precipitated by stressful events, such as participating in competitive sport or a musical performance, but can occur at rest in response to other stressful thoughts or events. The condition can exist as a comorbidity with asthma (and other pulmonary conditions) or may mimic asthma in individuals without asthma. The symptoms have a significant impact on quality of life, which has been shown to be more impaired than for those with asthma [8].

Clinical experience, and feedback from patients and their families, indicates that young people with dysfunctional breathing experience significant improvements in both physical health and quality of life following a course of physiotherapy where breathing retraining is the key element of therapy. Although little guidance is given, both the 2009 British Thoracic Society/Association of Chartered Physiotherapists in Respiratory Care "Guidelines for the physiotherapy management of the adult, medical, spontaneously breathing patient" [11] and the Global Initiative for Asthma guidelines suggest that dysfunctional breathing can be managed with breathing retraining or that breathing techniques may a useful supplementation to medication (Grade B) [12]. However, a recent Cochrane Review of dysfunctional breathing in children [13] demonstrates that no studies have been carried out investigating the value of breathing retraining for children and adolescents with dysfunctional breathing. As a result there is little or no evidence available to clinicians to guide assessment techniques, choose therapeutic interventions or assess outcomes; hence, little service provision is available for these patients.

In response to the clinical need for a dedicated clinic for children presenting with this condition, the BreathWorks clinic was established at Sheffield Children's Hospital in 2011 and is run by an experienced physiotherapist (N.B.). The rationale for the clinic was that a structured, but individualised, programme of physiotherapy interventions would improve clinical outcomes and quality of life for young people with dysfunctional breathing and that the improvement would be maintained following cessation of treatment. As the first clinic of its kind in the UK, it was felt important that its utility be assessed, and the data presented here were collected as part of a clinical evaluation of the service and its impact.

\section{Methods}

\section{Patients}

Patients were recruited from the BreathWorks clinic. The criteria for referral to the clinic are that patients have a clinical diagnosis of dysfunctional breathing made by an experienced Consultant or Specialist Registrar in Paediatric Respiratory Medicine, based on history highly suggestive of the condition with exclusion or optimal management of other conditions. Subjects were 7-16 years old at the time of referral. Patients were excluded from the study if they did not complete the course of treatment.

\section{Intervention}

All children received breathing retraining and education as their key interventions. The intervention was delivered by a specialist physiotherapist on a one-to-one basis with the active involvement of a parent, carer or other member of the family. The duration of the first session was up to $1.5 \mathrm{~h}$, with subsequent sessions lasting up to $1 \mathrm{~h}$.

The education component focussed on explaining the nature of the condition, sharing information on basic anatomy and the mechanics of breathing, while also giving the physiotherapist the opportunity to gauge the child's and family's level of knowledge and understanding. The breathing retraining method involved teaching diaphragmatic breathing. This was aimed at achieving nasal breathing, a normal respiratory rate, tidal volume, inspiratory/expiratory ratio, the feeling of control and a resultant return to a normal sigh/yawn rate, and a reduction in upper chest and accessory muscle use. Diaphragmatic breathing was first taught in supine and then progressed through sitting to more demanding and functional positions. The techniques were adjusted for children with the use of age-appropriate language and activities. 
Treatment programmes were also individualised based on the child's initial and ongoing assessment, and, where appropriate, included one or more of the following additional interventions: postural correction, thoracic mobility exercises, sniffs and vocal fricatives (for those with a laryngeal component), relaxation, shortness of breath management strategies and exercise advice. With the exception of sniffs and vocal fricatives (which are commonly used in speech and language therapy practice), all techniques used in the intervention are core physiotherapy techniques used with patients with many different respiratory conditions [4, 14-18]. The novel aspect of this study is applying them to this particular patient group.

\section{Outcome measures}

Quality of life was measured using the Pediatric Quality of Life Inventory (PedsQL), parent proxy and child report versions $[19,20]$, where the minimal clinically important difference for PedsQL child and PedsQL parent is 4.4 and 4.5 points, respectively [16]. Symptom score was measured using the Nijmegen Questionnaire [21, 22].

Each child and family completed quality of life and symptom questionnaires at the start of treatment (time point 1) and at the end of the course of treatment (time point 2). In order to determine whether the clinic was achieving long-term improvement the child and family were also asked to complete the questionnaires 6 months after the completion of treatment (time point 3). The questionnaires for the first two time points were completed in the clinic environment and the 6 month follow-up questionnaires were posted to the family.

Data analysis

Study data are presented as median (interquartile range (IQR)) to allow for some data not being normally distributed. The Wilcoxon signed rank test was used to compare data where only information from time points 1 and 2 were available. In the cases where data was available from all three time points, Freidman's test followed by Dunn's test was employed. The decision was made to use nonparametric tests for all data sets (including those that were normally distributed) to allow comparison of the various statistical analyses. All statistical analysis was performed using Prism version 6.02 (GraphPad, San Diego, CA, USA) and statistical significance was accepted as $\mathrm{p}<0.05$.

\section{Ethical approval}

Ethical approval was not required for this study as all children involved were receiving routine care, as provided by this institution, but approval was given by the Hospital Quality and Standards Department.

\section{Results}

During the study period 34 children were referred to the BreathWorks clinic. 21 subjects had asthma and the remaining 13 subjects did not have a comorbidity. The mean number of clinic visits was $n=4$ over an average period of 3 months. Seven subjects did not complete the intervention and no difference was apparent in terms of age, gender or presence/absence of comorbidity between children that did or did not complete the intervention. Time point 2 (discharge) parental questionnaires were not available for four subjects (as the children were brought to their final appointment by other family members).

Complete child and parent questionnaire data obtained prior to treatment (time point 1) and at discharge (time point 2) was therefore available for 23 children. 12 (52\%) of these were females and the cohort median (IQR) age was 13.3 (9.1-16.3) years. From time point 1 to time point 2, the median (IQR) PedsQL child score improved from 70.6 (55.4-77.1) to 84.7 (75.0-90.2), with the median (IQR) PedsQL parent score showing an even greater improvement from 58.6 (50.0-67.3) to 82.6 (72.8-90.2). For the same time period, the median (IQR) symptom score as measured by the Nijmegen Questionnaire decreased (demonstrating improvement) from $25.0(18.0-32.0)$ to 12.0 (8.0-17.0). These differences were all significant with $\mathrm{p}<0.0001$ (table 1). These results represent an improvement in symptom score and quality of life for $91 \%$ (21 of 23) of the children, with an improvement in the child's quality of life reported by $100 \%$ of parents/guardians.

10 families did not return their time point 3 postal questionnaires, leaving 13 children who completed all three sets of questionnaires. As with the cohort as a whole the data for these children demonstrated a significant difference in scores between time points 1 and 2 (table 2).

Importantly, there was no significant difference in the same scores (Nijmegen Questionnaire, PedsQL child and PedsQL parent) between time point 2 and time point 3 ( $p>0.05)$, indicating that the improvements were sustained up to 6 months following discharge from the clinic. Figure 1 shows summary data for each measure at each of the three time points.

\section{Discussion}

This study is the first to specifically investigate the short- and medium-term outcomes of breathing retraining for children with dysfunctional breathing. The results indicate that this intervention leads to a 


\section{TABLE 1 Data from completed questionnaires at time points 1 and 2}

\begin{tabular}{|c|c|c|c|c|}
\hline & \multirow[t]{2}{*}{ Subjects } & \multicolumn{2}{|c|}{ Score } & \multirow[t]{2}{*}{ p-value ${ }^{\#}$} \\
\hline & & Time point 1 & Time point 2 & \\
\hline PedsQL child & 23 & 70.60 (55.40-77.10) & $84.70(75.00-90.20)$ & $<0.0001$ \\
\hline PedsQL parent & 23 & $58.60(50.00-67.30)$ & $82.60(72.80-90.20)$ & $<0.0001$ \\
\hline Nijmegen Questionnaire & 23 & $25.00(18.00-32.00)$ & $12.00(8.00-17.00)$ & $<0.0001$ \\
\hline
\end{tabular}

statistically and clinically significant benefit in both quality of life and symptom scores. The results also indicate that these improvements are maintained for at least 6 months after the course of treatment has been completed. It should be noted that the improvements in the PedsQL child and PedsQL parent of 14.1 and 24 are considerably greater than the values deemed to represent the established minimal clinically important differences of 4.4 and 4.5 , respectively.

Our results are consistent with the one randomised controlled trial in adults in which breathing retraining for the management of dysfunction breathing was assessed, which also found significant improvements in symptom scores and quality of life [15]. However, the natural history of the condition has not been studied in detail and hence it is not known what the outcome for these children would have been had they not been exposed to the intervention. A report from the early 1980s found, in a cohort of children and adolescents with the condition in whom there was no intervention other than reassurance, that symptoms persisted in $40 \%$ of cases into adulthood [23]. In comparison, the current study indicates that the outcomes following active intervention are much improved.

In this study, physiotherapy intervention was studied in isolation as this is the model of care currently used at Sheffield Children's National Health Service Foundation Trust. The BreathWorks clinic is run by a specialist respiratory physiotherapist, and the results reflect the impact of breathing retraining and associated interventions which include, where appropriate, relaxation techniques. These subjects are not routinely referred to a psychologist as, in our experience, the vast majority of subjects with thoracic or extrathoracic dysfunctional breathing do not have significant psychological issues. Early reports in the literature did suggest that significant psychological distress was frequently associated with pronounced symptoms of dysfunctional breathing, but subsequent experience suggests that psychological intervention is required in only a minority of subjects [24]. In future studies of the condition, it would be interesting to explore whether a psychotherapeutic intervention would augment the outcomes of treatment or provide an alternative for subgroups of patients.

As we anticipated, a significant proportion of subjects in our cohort had asthma as a comorbidity and were receiving inhaled asthma medications at the start of the study. We did not record inhaler usage or other healthcare utilisation data prospectively in this study, but plan to do so in future studies since patients anecdotally reported being less reliant on their reliever medication. Hence, the impact of the intervention on use of medication and other healthcare resources needs to be explored.

Research in dysfunctional breathing, however, remains problematic in that there are no clear diagnostic criteria that can be applied, potentially leading to heterogeneity in study populations. There are also no condition-specific validated outcome measures currently available. The generic scales of the PedsQL were

TABLE 2 Data from completed questionnaires at time points 1, 2 and 3

\begin{tabular}{|c|c|c|c|c|c|}
\hline & \multirow[t]{2}{*}{ Subjects } & \multicolumn{3}{|c|}{ Score } & \multirow[t]{2}{*}{ p-value ${ }^{\#}$} \\
\hline & & Time point 1 & Time point 2 & Time point 3 & \\
\hline PedsQL child & 13 & $76.00(51.60-81.30)$ & 89.10 (82.60-91.30) & 85.80 (79.30-91.30) & $<0.001$ \\
\hline PedsQL parent & 13 & $65.20(56.50-71.70)$ & 89.10 (78.80-91.30) & 81.50 (77.70-95.10) & $<0.001$ \\
\hline Nijmegen Questionnaire & 13 & $18.00(15.00-30.50)$ & $11.00(5.00-15.50)$ & $8.00(6.50-15.00)$ & $<0.003$ \\
\hline
\end{tabular}



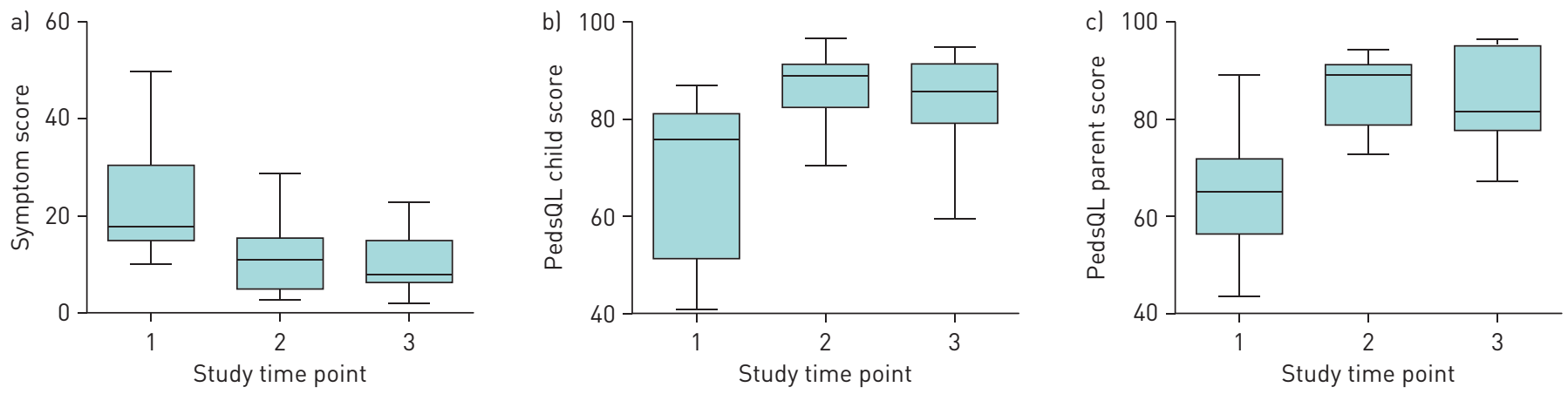

FIGURE 1 Summary data for a) symptom score (Nijmegen Questionnaire), b) child-rated quality of life score and c) parent-rated quality of life score at each of the three time points $(n=13)$. PedsQL: Pediatric Quality of Life Inventory.

chosen as they are well validated $[19,20]$, but no validated measure of symptoms is available. The Nijmegen Questionnaire is the most widely used "objective" tool, but there are a number of problems with the use of a tool developed for use in adults with a view to identifying the "hyperventilation syndrome" [18, 19]. In particular, it does not appear to capture symptoms specifically attributable to "hyperventilation". Many of the symptoms included in the questionnaire (e.g. chest pain) are probably related to dysfunctional breathing rather than hypocapnoea in most subjects, while many are probably not directly related to dysfunctional breathing. One study [25] characterised the questions included in the questionnaire under the categories of tension, peripheral neurovascular, central neurovascular and dyspnoea. The authors found that the dyspnoea subscore of the Nijmegen Questionnaire correlated closely with a manual assessment of dysfunctional breathing. Moreover, they observed that it was those subjects with significant scores on the dyspnoea subscores who were found to benefit most from breathing retraining and this was more predictive of a response than the overall score on the Nijmegen Questionnaire.

The issue of specificity of questionnaires was also highlighted in a report of children whom in which low scores on the Childhood Asthma Control Test led to the inappropriate use of high doses of asthma medication in children with dysfunctional breathing [26]. These observations reflect the significant overlap between symptoms associated with dysfunctional breathing and those due to primary respiratory conditions such as asthma which make the design of an entirely specific questionnaire problematic. In the context of this study, however, the Nijmegen Questionnaire provides a tool to assess the impact of the intervention on symptoms experienced by the subjects, and it is reassuring to note that the changes mirrored the improvements in quality of life reported by the subjects and their parents.

The lack of a condition-specific questionnaire also inhibited the collection of detailed data able to show change in specific symptoms or explanations for susceptibility to change of certain symptoms. Clinical experience with these patients was that symptoms commonly improve in parallel with each other, but we could not explore this scientifically. In addition, the numbers of patients involved in the study were too small to gain representative information from a subgroup analysis with regard to comorbidities. We plan to develop a condition-specific questionnaire in the future.

The reason for some patients not attending follow-up clinic appointments was not explored as the study was not designed to address this. However, there were no differences in the demographic data of those who completed their visits and those who did not. It is likely that a variety of practical considerations, such as having to take time off work for parents and school, contributed to some of the nonattendance. While the return of questionnaires at 6 months post-completion of the intervention was only $57 \%$ of those returned at discharge, this was higher than the anticipated rate based on the published data for this type of survey [27] and no specific features for those families that did not return their questionnaires were identified.

The results from this study suggest that breathing retraining appears to offer significant benefits for children and adolescents with dysfunctional breathing both in terms of quality of life and symptoms experienced. The data supports the need for well-designed prospective clinical trials to investigate the short-, medium- and long-term effects of this intervention, with a view to optimising treatment and ameliorating the significant morbidity experienced by children with dysfunctional breathing.

\section{References}

1 Barker N, Everard ML. Getting to grips with 'dysfunctional breathing'. Paediatr Respir Rev 2015; 15: 53-56.

2 Courtney R. The functions of breathing and its dysfunctions and their relationship to breathing therapy. Int $J$ Osteopathic Med 2009; 12: 78-85. 
Peper E, Tibbetts V. Effortless diaphragmatic breathing. Physical Therapy Products 1994; 6: 67-71.

Clifton-Smith T, Rowley J. Breathing pattern disorders and physiotherapy. Phys Ther Rev 2011; 16: 75-86.

de Groot EP. Breathing abnormalities in children with breathlessness. Paediatr Respir Rev 2011; 12: 83-87.

Noyes BE, Kemp JS. Vocal cord dysfunction in children. Paediatr Respir Rev 2007; 8: 155-163.

Maturo S, Hill C, Bunting G, et al. Pediatric paradoxical vocal-fold motion: presentation and natural history. Pediatrics 2011; 128: e1443-e1449.

8 Thomas M, McKinley RK, Freeman E, et al. Prevalence of dysfunctional breathing in patients treated for asthma in primary care: cross sectional survey. BMJ 2001; 322: 1098-1100.

9 Thomas M, McKinley RK, Freeman E, et al. The prevalence of dysfunctional breathing in adults in the community with and without asthma. Prim Care Respir J 2005; 14: 78-82.

10 de Groot E, Duiverman E, Brand P. Dysfunctional breathing in children with asthma: a rare but relevant comorbidity. Eur Respir J 2013; 41: 1068-1073.

11 Bott J, Blumenthal S, Buxton M, et al. Guidelines for the physiotherapy management of the adult, medical, spontaneously breathing patient. Thorax 2009; 64: i1-i51.

12 Global Initiative for Asthma. GINA Report: Global Strategy for Asthma Management and Prevention. www.ginasthma.org/local/uploads/files/GINA_Report_2015.pdf Date last accessed: August 12, 2015.

13 Barker NJ, Jones M, O'Connell NE, et al. Breathing exercises for dysfunctional breathing/hyperventilation syndrome in children. Cochrane Database Syst Rev 2013; 12: CD010376.

14 Holloway EA, West RJ. Integrated breathing and relaxation training (the Papworth method) for adults with asthma in primary care: a randomised controlled trial. Thorax 2007; 62: 1039-1042.

15 Chaitow L, Bradley D, Gilbert C. Multidisciplinary Approaches to Breathing Pattern Disorders. London, Churchill Livingstone, 2002.

16 Jones M, Troup F, Nugus J, et al. Does manual therapy provide additional benefit to breathing retraining in the management of dysfunctional breathing? A randomised controlled trial. Disabil Rehabil 2015; 37: 763-770.

17 Grammatopoulou EP, Skordilis EK, Stavrou N, et al. The effect of physiotherapy-based breathing retraining on asthma control. J Asthma 2011; 48: 593-601.

18 Hagman C, Janson C, Emtner M. Breathing retraining - a five-year follow-up of patients with dysfunctional breathing. Respir Med 2011; 105: 1153-1159.

19 Varni JW, Seid M, Kurtin PS. PedsQL ${ }^{\mathrm{TM}}$ 4.0: reliability and validity of the Pediatric Quality of Life Inventory ${ }^{\mathrm{TM}}$ version 4.0 generic core scales in healthy and patient populations. Med Care 2001; 398: 800-812.

20 Varni JW, Burwinkle TM, Seid M, et al. The PedsQL ${ }^{\mathrm{TM}} 4.0$ as a pediatric population health measure: feasibility, reliability, and validity. Ambul Pediatr 2003; 3: 329-341.

21 van Dixhoorn J, Duivenvoorden HJ. Efficacy of Nijmegen Questionnaire in recognition of the hyperventilation syndrome. J Psychosom Res 1985; 29: 199-206.

22 van Doorn P, Folgering $\mathrm{H}$, Colla P. Control of the end-tidal $\mathrm{PCO}_{2}$ in the hyperventilation syndrome: effects of biofeedback and breathing instructions compared. Bull Eur Physiopathol Respir 1982; 18: 829-836.

23 Herman S, Stickler G, Lucas A. Hyperventilation syndrome in children and adolescents: long-term follow-up. Pediatrics 1981; 67: 183-187.

24 Depiazzi J, Everard ML. Dysfunctional breathing and reaching one's physiological limit as significant causes of exercise induced dyspnoea - the importance of accurate assessment. Breathe 2016; 12: 120-129.

25 Courtney R, van Dixhoorn J, Greenwood KM, et al. Medically unexplained dyspnea: partly moderated by dysfunctional (thoracic dominant) breathing pattern. J Asthma 2011; 48: 259-265.

26 Peroni DG, Piacentini GL, Bodini A, et al. Childhood Asthma Control Test in asthmatic children with dysfunctional breathing. J Allergy Clin Immunol 2008; 121: 266-267.

27 Iglesias C, Torgerson D. Does length of questionnaire matter? A randomised trial of response rates to a mailed questionnaire. J Health Serv Res Policy 2000; 5: 219-221. 